\title{
QTL Analysis of Fusarium Head Blight Severity in Recombinant Inbred Population Derived from a Cross between Two-rowed Barley Varieties
}

\author{
Kiyosumi Hori, Kazuhiro Sato*, Tetsuro Kobayashi and Kazuyoshi Takeda \\ Research Institute for Bioresources, Okayama University, 2-20-1 Chuo, Kurashiki, Okayama 710-0046, Japan
}

\begin{abstract}
Fusarium head blight (FHB) severity was evaluated in a set of recombinant inbred (RI) lines from a cross between the two-rowed barley varieties, Harbin 2-row (resistant) and Turkey 6 (susceptible). Harbin 2-row was one of the most resistant varieties to FHB among ca. 5,000 barley germplasm accessions stored at Okayama University. The FHB severity of the RI lines and their parents was evaluated by the 'cut-spike test' in the 2000-2001 and 2003-2004 seasons. Spikes with disease symptoms were scored based on eleven grades from resistant (0) to susceptible (10). A high-density linkage map, including 328 AFLP, 45 SSR, one RFLPSTS, one AFLP-STS, four resistance gene analog (RGA) and four morphological markers, was constructed to detect QTLs for FHB severity. Composite interval mapping enabled to detect one QTL on chromosome $2 \mathrm{H}$ in the 2000-2001 season, and one QTL on chromosome $4 \mathrm{H}$ and another QTL on chromosome $6 \mathrm{H}$ in the 2003-2004 season. At these three QTLs, the alleles from Harbin 2-row contributed to a lower severity. A QTL on chromosome $2 \mathrm{H}$ was located in the vicinity of the cleistogamy locus (cly1 or Cly2) that determines open/closed flowering type. The comparison with previously identified QTLs for FHB severity by the present authors indicated that Harbin 2-row might harbor the same resistance allele in the vicinity of the $c l y 1 / C l y 2$ locus as Russia 6, which was used as a resistant parent in the previous study. The QTL in the vicinity of the cly $1 /$ Cly2 locus is one of the promising sources for FHB resistance breeding in barley.
\end{abstract}

Key Words: Hordeum vulgare L., barley, Fusarium head blight, quantitative trait loci, cleistogamy.

\section{Introduction}

Fusarium head blight (FHB) or scab, which is one of the most serious diseases of barley, causes significant losses in both yield and quality (Atanasoff 1920, Ayoub and Mather 2002, Steffenson 2003). Fusarium infected-kernels often contain high concentrations of mycotoxins, which are harmful to human beings and livestock (Bai and Shaner 1994). The cultivation of resistant plants is the most costeffective method to control the disease (Mesfin et al. 2003).

QTL analyses associated with FHB severity and deoxynivalenol (DON) accumulation have been conducted in barley to select resistant genotypes, and identify the responsible genetic factors (de la Pena et al. 1999, Zhu et al. 1999, Ma et al. 2000, Mesfin et al. 2003, Dahleen et al. 2003). In previous studies, some associations between FHB resistance and certain morphological traits had also been reported. For example, several extensive germplasm screening studies indicated that two-rowed varieties ( Vrs 1 ) showed a higher FHB resistance than six-rowed (vrs l) varieties (Takeda and Heta 1989, Zhou et al. 1991, Steffenson et al. 1996). Mesfin et al. (2003) and Dahleen et al. (2003) reported the presences of a QTL near the vrs 1 locus on chromosome $2 \mathrm{H}$ using

Communicated by Y. Takahata

Received March 28, 2005. Accepted October 5, 2005.

*Corresponding author (e-mail: kazsato@rib.okayama-u.ac.jp) populations derived from two-rowed $\times$ six-rowed crosses. They could not determine whether the QTL effect was caused by the pleiotropic effect of the vrs 1 locus or the linkage between the QTL and the vrs 1 locus. The QTL at the vrs 1 locus was also detected in a recombinant inbred (RI) population derived from a cross between Russia 6 (tworowed) and H.E.S. 4 (six-rowed) (Hori et al. 2005).

Steffenson (2003) and Yoshida et al. (2005) reported the presence of relationship between the flowering type (anther exsertion or cleistogamy) and FHB resistance. The gene for the flowering type was identified as a morphological marker (cly1/Cly2) on the long arm of chromosome 2H (Turuspekov et al. 2004). In our previous study (Hori et al. 2005), we showed that the lines with closed flowering (without anther exsertion) were significantly more resistant than the lines with open flowering, and a QTL for FHB severity was detected within a $1.7 \mathrm{cM}$ distance from the $c l y 1 / \mathrm{Cly} 2$ locus. Further analysis is necessary to reveal the relationship between the severity and the responsible gene(s) in this region.

The objectives of the present study were to estimate the locations and effects of QTLs for FHB severity on a linkage map in a RI population derived from a cross between Harbin 2-row (two-rowed and FHB-resistant variety) and Turkey 6 (two-rowed and FHB-susceptible variety), and to examine whether these QTLs exist in the vicinity of the vrs 1 or clyl/ Cly2 loci. The locations and effects of QTLs for FHB severity detected in the present study were compared with those in 
previous studies to eliminate the row-type effects, using a RI population derived from a cross between varieties with the same row type.

\section{Materials and Methods}

\section{Plant materials}

Two hundred thirty five $\mathrm{F}_{12}$ RI lines derived from a cross between Harbin 2-row and Turkey 6 were developed by single-seed descent from $\mathrm{F}_{2}$ individuals in a field located at the Research Institute for Bioresources, Okayama University, Kurashiki, Japan $\left(34^{\circ} 35^{\prime} \mathrm{N}\right.$ and $\left.133^{\circ} 46^{\prime} \mathrm{E}\right)$. Harbin 2row (accession No. C649), which is a two-rowed spring barley variety collected from China, was selected as one of the most resistant varieties to FHB by Takeda and Heta (1989). Turkey 6 (accession No. T602) is a moderately susceptible two-rowed winter barley variety collected from Turkey. Ninety-four RI lines were used to construct a linkage map and to identify QTLs for FHB severity.

\section{Phenotype evaluation}

The RI lines and their parents were grown in a field located in Kurashiki during the winter barley growing seasons of 2000-2001 (2001 season) and 2003-2004 (2004 season). FHB inoculation was carried out by the 'cut-spike test' developed by Takeda and Heta (1989). Spikes from each line were collected from the field at the time of flowering, and spikes were spray-inoculated with a freshly prepared conidial suspension (concentration of $2 \times 10^{5}$ per ml) at the rate of $50 \mathrm{ml}$ per inoculation tray (ca. 240-300 spikes). Fusarium graminearum strain OUZ78, which displays a moderate virulence and is maintained at the Research Institute for Bioresources, Okayama University, was used as a source of inoculum. Spikes with disease symptoms were scored on the eighth day after inoculation using a 0-10 scale, where 0 corresponded to $0 \%$ of infected florets per spike (resistant), and 10 to over $60 \%$ of infected florets per spike (susceptible). The experiment consisted of three replications with three inoculated spikes in each replication in both seasons. The mean scores of replications for each RI line were used for QTL analysis.

Thirteen qualitative and quantitative traits were evaluated for the 94 RI lines and their parents. Lemma color, aleurone-layer color, rachilla-hair length and flowering type (cleistogamy) were scored and mapped as morphological markers on a linkage map of the RI population. In the 2001 season, phenotypes were scored for nine quantitative traits, including culm length, ear length, spike-exsertion length (culm length between base of spike and flag leaf), awn length, number of spikelets per spike, rachis-internode length (kernel density), glume length, thousand-kernel weight and heading date. Scorings of quantitative traits were performed according to the method of Hori et al. (2003). Heading date was scored in both the 2001 and 2004 seasons. Phenotypes for the agronomic traits of Harbin 2-row and Turkey 6 are shown in Table 1.
Table 1. Phenotypes of Harbin 2-row and Turkey 6 for four morphological and nine quantitative traits

\begin{tabular}{lcc}
\hline \hline Trait & Harbin 2-row & Turkey 6 \\
\hline Lemma color & Normal & Black \\
Aleurone-layer color & Normal & Blue \\
Rachilla-hair length & Long & Short \\
Flowering type & Closed & Open \\
Culm length (cm) & 112.3 & 69.8 \\
Ear length (cm) & 10.1 & 8.4 \\
Spike-exsertion length (cm) & 21.7 & 5.7 \\
Thousand-kernel weight (g) & 58.8 & 41.6 \\
Awn length (cm) & 18.9 & 13.1 \\
Glume length (mm) & 14.0 & 9.7 \\
Number of spikelets/spike & 33.3 & 23.3 \\
Rachis-internode length (mm) & 2.7 & 2.9 \\
Heading date (2001) (day from April 1) & 28.0 & 28.0 \\
Heading date (2004) (day from April 1) & 21.0 & 20.0 \\
\hline
\end{tabular}

\section{Linkage map construction}

Each 94 RI line was genotyped with AFLP, SSR, RFLP-STS, AFLP-STS, RGA and morphological markers scattered on the whole genome. AFLP markers were analyzed based on the description by Vos et al. (1995). SSR markers generated by Ramsay et al. (2000) were amplified, as described by Hori et al. (2003). One RFLP-STS marker (cMWG699) closely located to the vrs 1 locus on chromosome $2 \mathrm{H}$ was selected to indicate the vrs 1 location in the present study, and amplified as described by Tanno et al. (2002). One AFLP-STS marker (STS_FEgtaMacg677) developed by Hori et al. (2005) was also used to compare QTL locations. Resistance gene analog (RGA) markers were amplified with XLRRfor and XLRRrev primers. For the primer sequences and amplification conditions of the RGA markers, the method of Chen et al. (1998) was adopted. The PCR products were electrophoresed on a $13 \%$ acrylamide gel at a $250 \mathrm{~V}$ constant voltage for seven hours using the High Efficiency Genome Scanning system (Hori et al. 2003).

The segregation data of AFLP, SSR, RFLP-STS, AFLP-STS, RGA and morphological markers were analyzed with MAPMAKER/EXP ver. 3.0 (Lander et al. 1987) and MAPL98 (Ukai et al. 1995). The SSR and RFLP-STS markers were used as anchors to assign other markers to the seven barley linkage groups at the LOD threshold of 3.0. The Kosambi mapping function was used to estimate marker distances (Kosambi 1944).

\section{QTL identification}

QTL analysis was carried out by simple interval mapping (SIM) and by composite interval mapping (CIM), using the software packages MAPMAKER/QTL ver. $1.1 \mathrm{~b}$ (Lander and Botstein 1989) and QTL Cartographer ver. 1.16 (Basten et al. 1994), respectively. A LOD threshold of 2.0, which was determined by the permutation test using QTL Cartographer, was used to confirm the presence of putative QTLs. The LOD peaks at significant QTL intervals were considered to correspond to the QTL locations on the linkage map. 


\section{Results}

\section{Evaluation of FHB resistance and agronomic traits}

Figure 1 shows the scores of 93 RI lines and the parents for FHB severity in the 2001 and 2004 seasons, displayed as a scatter diagram. FHB severity score of one RI line was not determined in the 2004 season. Harbin 2-row showed lower FHB severity scores than Turkey 6 in both seasons. The RI lines differed considerably in their FHB severity scores, ranging from 2.0 (resistant) to 9.3 (susceptible) in the 2001 season, and from 2.3 to 9.7 in the 2004 season. Broad sense heritabilities estimated from the analysis of variance in the RI lines (Falconer 1989) were 0.77 in the 2001 season and 0.74 in the 2004 season. The correlation coefficient for severity scores in the RI lines between the 2001 and 2004 seasons was 0.53 and significant at the $1 \%$ level (Fig. 1).

Correlation coefficients were calculated between the FHB severity score and nine agronomic traits. Heading date in the 2004 season $(r=0.26)$, ear length $(r=0.21)$, glume length $(r=-0.28)$ and rachis-internode length $(r=0.29)$ were significantly correlated with the severity score, although all the $r$-values were less than 0.30. Ear length $(r=0.21)$ and rachis-internode length $(r=0.29)$ showed significant positive correlations with the severity score, indicating a higher susceptibility in the RI lines with lax spikes. Glume length $(r=$ -0.28 ) showed a significant negative correlation with the severity score, indicating the presence of a higher resistance in the RI lines with short glumes. Heading date in the 2001

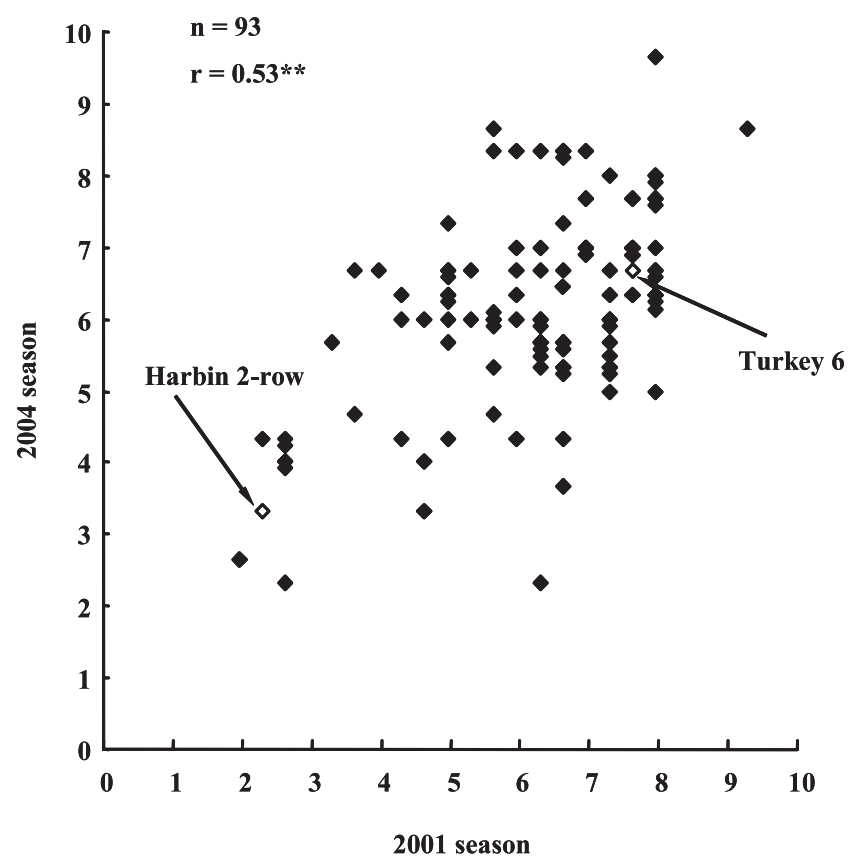

Fig. 1. Scatter diagram of FHB severity scores in the RI population derived from Harbin 2-row $\times$ Turkey 6 in the 2001 and 2004 seasons. FHB severity score 0 corresponds to $0 \%$ of infected florets per spike (resistant), and FHB score 10 to over $60 \%$ of infected florets per spike (susceptible). ${ }^{* *}$ : significance at the $1 \%$ level. season, culm length, awn length, spike-exsertion length, number of spikelets per spike and thousand-kernel weight were not significantly correlated with the severity score.

\section{Map construction}

Seventy-nine primer combinations in the AFLP analysis generated 426 polymorphic bands between Harbin 2-row and Turkey 6. Seventy-seven primer combinations of SSRs for parental screening generated 45 polymorphic markers. Both RFLP-STS (cMWG699) and AFLP-STS (STS_FEgtaMacg677) markers revealed single nucleotide polymorphisms between the parents. The XLRRfor and XLRRrev primer combinations generated four polymorphic fragments as RGA markers.

The final linkage map consisted of 383 markers including 328 AFLP, 45 SSR, one RFLP-STS, one AFLP-STS, four RGA and four morphological markers, with a total map length of $1,377.1 \mathrm{cM}$. The average marker density was 3.6 $\mathrm{cM} /$ locus. However, the marker density varied with the regions. Marker clusters were detected on each linkage group. In contrast, regions with few markers were observed on chromosomes $1 \mathrm{H}$ and $4 \mathrm{H}$. Most of the locations of the SSR markers were not different from those previously reported (Ramsay et al. 2000, Hori et al. 2003). cMWG699, which was close to the vrs 1 locus, was located on the long arm of chromosome $2 \mathrm{H}$. The lemma color $(B l p)$, flowering type (cly1/Cly2), aleurone-layer color (blx) and rachilla-hair length $(s r h)$ markers were located on chromosomes $1 \mathrm{H}, 2 \mathrm{H}$, $4 \mathrm{H}$ and $5 \mathrm{H}$, respectively. The locations of these morphological markers corresponded to their previously reported (Costa et al. 2001, Hori et al. 2003).

\section{QTL detection}

QTL analyses were carried out for FHB severity on the linkage map using MAPMAKER/QTL ver. 1.1b (SIM) and QTL Cartographer ver. 1.16 (CIM). Table 2 shows the characteristics of the detected QTLs in relation to the FHB severity, and Figure 2 shows the LOD score profiles by CIM analysis for FHB severity on chromosomes $2 \mathrm{H}, 4 \mathrm{H}$ and $6 \mathrm{H}$. No QTLs were detected by SIM analysis in either seasons. One QTL (LOD > 2.0) on chromosome $2 \mathrm{H}$, which was detected in the 2001 season, was located between the FXLRRfor_XLRRrev119 and STS_FEgtaMacg677 markers in the vicinity of the $c l y 1 / C l y 2$ locus. In the 2004 season, one QTL was detected each on chromosomes $4 \mathrm{H}$ and $6 \mathrm{H}$. All the QTL alleles from the resistant parent Harbin 2-row contributed to a lower FHB severity.

Non-significant LOD peaks $(<2.0)$ were observed at the same marker intervals as those of the QTLs detected on chromosomes $4 \mathrm{H}$ and $6 \mathrm{H}$ in the 2001 season and on chromosome $2 \mathrm{H}$ in the 2004 season (Table 2 and Fig. 2). These low LOD peaks showed almost similar additive effects (ca. 1.0) to those of the three significant QTLs (LOD>2.0). Multiple QTL model (MQM) with these three LOD peaks in the CIM analysis explained $26.2 \%$ of the total variance in the 2001 season and $38.4 \%$ in the 2004 season (Table 2). The MQM effect 
Table 2. QTLs associated with FHB resistance on the linkage map detected by composite interval mapping (CIM) and multiple QTL model (MQM)

\begin{tabular}{|c|c|c|c|c|c|c|}
\hline Chromosome & Marker interval & Year & $\begin{array}{l}\text { Position }^{1)} \\
\text { (cM) }\end{array}$ & $\mathrm{LOD}^{2)}$ & Var. $^{3)}(\%)$ & Weight $\left.{ }^{4}\right)$ \\
\hline \multirow[t]{2}{*}{$2 \mathrm{H}$} & FXLRRfor_XLRRrev119-STS_FEgtaMacg677 & 2001 & 0.2 & 2.6 & 10.1 & -1.3 \\
\hline & & 2004 & 0.2 & 1.5 & 7.3 & -0.8 \\
\hline \multirow[t]{2}{*}{$4 \mathrm{H}$} & FMacgEcgt288-HVM67 & 2001 & 2.6 & 1.9 & 9.1 & -0.9 \\
\hline & & 2004 & 0.3 & 2.2 & 15.5 & -0.9 \\
\hline \multirow[t]{2}{*}{$6 \mathrm{H}$} & FMataEagc408-HVM11 & 2001 & 6.9 & 1.9 & 8.7 & -0.9 \\
\hline & & 2004 & 6.1 & 2.7 & 18.6 & -1.0 \\
\hline \multirow[t]{2}{*}{ Total } & (MQM) & 2001 & & & 26.2 & \\
\hline & & 2004 & & & 38.4 & \\
\hline
\end{tabular}

1) Distance of peak LOD score position from the left side marker

2) Peak LOD score.

3) Explained variance.

4) Estimated additive effect of Harbin 2-row allele.

was almost equal to the sum of additive effects of each QTL, indicating that interactions among the QTLs were limited.

Two QTLs were detected on chromosome $2 \mathrm{H}$ in a RI population derived from a cross between Russia 6 and H.E.S. 4 (Hori et al. 2005). Both varieties, Russia 6 and Harbin 2-row, displayed the highest level of resistance to FHB, as reported by Takeda and Heta (1989). Thus, QTL locations on chromosome $2 \mathrm{H}$ were compared between the two linkage maps (Fig. 2). Marker orders including HVM54, FXLRRfor_XLRRrev119 (170), STS_FEgtaMacg677 and clyl/Cly2 loci were identical between the linkage maps of Harbin 2-row $\times$ Turkey 6 and Russia $6 \times$ H.E.S. 4 . The QTL for FHB severity was located at the same marker intervals in the vicinity of the $\mathrm{cly} 1 / \mathrm{Cly} 2$ locus between the linkage maps. No QTL was detected in the vicinity of the vrs 1 (cMWG699) locus in the Harbin 2-row $\times$ Turkey 6 linkage map.

\section{Discussion}

\section{Linkage map construction}

A linkage map including 383 loci was constructed for the RI population derived from the cross between Harbin 2row and Turkey 6 in the present study. Length of the linkage map was $1,377.1 \mathrm{cM}$, which seemed to cover the whole genome, because a sufficiently large number of markers was distributed on the seven barley chromosomes. Thus, the linkage map in the present study may be suitable for the detection of QTLs for FHB severity. The total map length was almost the same as that of the map constructed by Costa et al. $(2001 ; 1,387 \mathrm{cM})$, longer than that of Ramsay et al. $(2000 ; 1,173 \mathrm{cM})$ and shorter than that of Hori et al. (2003; $1,595.7 \mathrm{cM})$. The difference in the length of various maps may be due to differences in the recombination frequencies in the cross combinations and population types of generations, i.e. $\mathrm{F}_{2}$, RI or doubled haploid.

\section{QTL analysis for FHB resistance}

Broad sense heritabilities were relatively high for the FHB severity $\left(h^{2}=0.77\right.$ in the 2001 season and $h^{2}=0.74$ in the 2004 season). The correlation coefficient between the two seasons was significant at the $1 \%$ level $(r=0.53)$. However, the LOD peaks were only significant $(>2.0)$ on chromosome $2 \mathrm{H}$ in the 2001 season and on chromosomes $4 \mathrm{H}$ and $6 \mathrm{H}$ in the 2004 season (Table 2 and Fig. 2). Total explained variances of the three LOD peaks were $26.2 \%$ in the 2001 season and $38.4 \%$ in the 2004 season (Table 2). It was suggested that some of the QTLs for FHB severity with smaller explained variances were not detected in the present study. Improvements of phenotype scoring, including plant cultivation in a controlled environment, would be necessary to detect a larger number of QTLs in this RI population.

No QTL for FHB severity was detected in the vicinity of the vrs 1 (cMWG699) locus in the present study, presumably due to the presence of the same resistance alleles around the vrs 1 locus between Harbin 2-row and Turkey 6. In our previous study using a two-rowed $\times$ six-rowed RI population, one QTL for FHB severity located at the vrs 1 locus was detected in only one season, although the other QTLs for FHB severity were detected in both seasons (Hori et al. 2005; Fig. 2). Mesfin et al. (2003) and Dahleen et al. (2003) also reported the presence of QTLs for FHB severity in the vicinity of the vrs 1 locus on chromosome $2 \mathrm{H}$ in two-rowed $\times$ six-rowed populations. Thus, the $v r s 1$ locus might exert a pleiotropic effect conferring FHB resistance, but may not be the major factor for FHB resistance.

It has been suggested that the flowering type (anther exsertion or cleistogamy) influenced FHB resistance (Steffenson 2003, Turuspekov et al. 2004, Yoshida et al. 2005). In our previous study, we showed that a QTL for FHB severity was detected within a $1.7 \mathrm{cM}$ distance from the cly1/Cly2 locus. This QTL exerted the largest effect in the Russia $6 \times$ H.E.S. 4 population (Fig. 2). In the present study, one QTL was also detected in the vicinity of the $\mathrm{cly} 1 / \mathrm{Cly} 2$ locus $(5.8 \mathrm{cM}$ distance). Since several RI lines with open flowering showed a high level of FHB resistance, while several RI lines with closed flowering showed a low level of FHB resistance, the possibility of linkage cannot be ruled out. Moreover, multiple QTLs for FHB severity were reported in 
Chromosome 2H

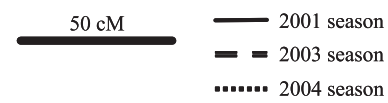

(Harbin 2-row $\times$ Turkey 6)
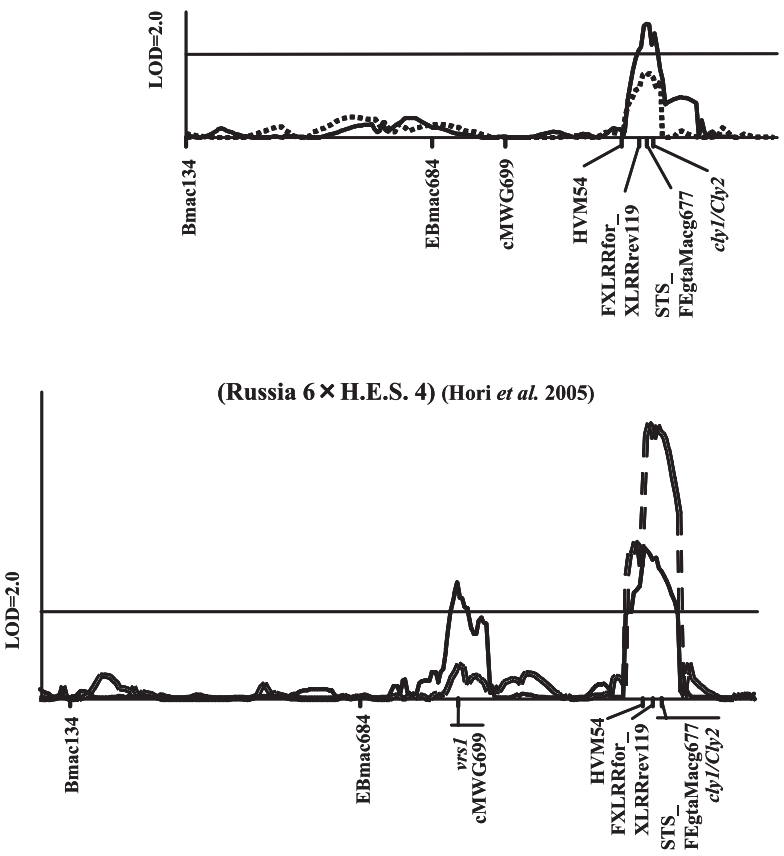

Chromosome 4H

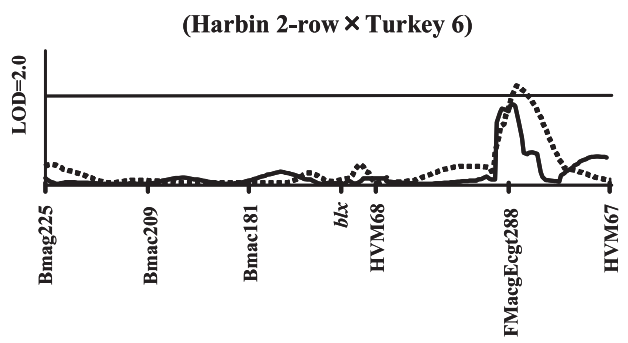

Chromosome 6H

(Harbin 2-row $\times$ Turkey 6)

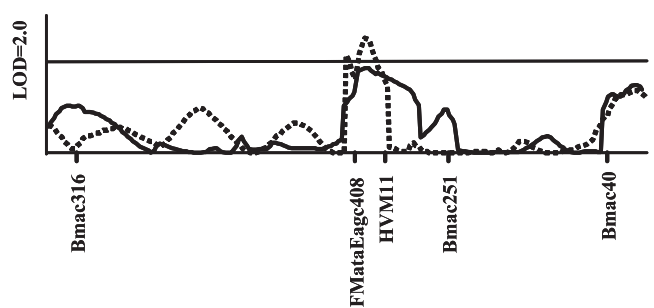

Fig. 2. Scans of QTL analysis (composite interval mapping) for FHB severity on chromosomes $2 \mathrm{H}, 4 \mathrm{H}$ and $6 \mathrm{H}$ on the linkage map in the RI population derived from Harbin 2-row $\times$ Turkey 6 , and chromosome $2 \mathrm{H}$ on the linkage map in the RI population derived from Russia $6 \times$ H.E.S. 4 (Hori et al. 2005). Linkage groups are oriented from short arm (left) to long arm (right). Locations and designations of anchor markers and closely linked markers to the QTL are shown on horizontal lines under the LOD curves. this chromosomal region in several previous studies (de la Pena et al. 1999, Zhu et al. 1999, Ma et al. 2000, Mesfin et al. 2003, Dahleen et al. 2003). Further studies using largescale populations or near-isogenic lines should be carried out to determine whether the resistance is derived from the linkage effect of the cly1/Cly2 locus.

This is the first report of QTL detection for FHB severity on the long arm of chromosome 4H (Fig. 2), although de la Pena et al. (1999) and Ma et al. (2000) detected several QTLs for FHB severity on the short arm of chromosome 4H. Another QTL was detected near the centromeric region on chromosome 6H (Fig. 2). Ma et al. (2000), Mesfin et al. (2003) and Dahleen et al. (2003) reported the presence of QTLs for FHB on chromosome 6H. Some of the reported QTLs may correspond to the QTL detected in the present study.

Genetic relationships between FHB severity and nine agronomic traits were investigated. Low but statistically significant correlations with FHB severity were detected for ear length $(r=0.21)$, glume length $(r=-0.28)$ and rachisinternode length $(r=0.29)$ in the 2001 season. Since QTLs for FHB severity, ear length, glume length and rachis-internode length were identified in the vicinity of the $c l y 1 / C l y 2$ locus on chromosome $2 \mathrm{H}$ (data not shown), significant correlations might have resulted from the linkage between the QTL for FHB severity and the QTLs for these traits. A low but statistically significant correlation with FHB severity was also detected for heading date $(\mathrm{r}=0.26)$ in the 2004 season. Because one QTL for heading date was detected at a $12.5 \mathrm{cM}$ distance from the QTL for FHB severity on chromosome $6 \mathrm{H}$ in the 2004 season (data not shown), the low but significant correlation in the 2004 season might be due to linkage between the QTLs for heading date and FHB severity. Culm length, awn length, spike-exsertion length, number of spikelets per spike and thousand-kernel weight did not show any significant correlations with FHB severity, and QTLs for these traits were not detected near the QTLs for FHB severity (data not shown). Thus, FHB severity detected by the 'cut-spike test' seemed independent from these traits in the present study.

Since the markers linked with the QTLs are important to perform marker-assisted selection for FHB resistance, the RI lines were selected based on the genotypes of the QTL flanking markers and average FHB scores were compared to demonstrate the multiple effects of the FHB resistance alleles. FHB severity scores of selected RI lines with three Harbin 2-row QTL alleles and those with three Turkey 6 QTL alleles were $5.3 \pm 1.4$ and $7.3 \pm 1.3$ in the 2001 season, and $4.8 \pm 1.5$ and $7.0 \pm 1.3$ in the 2004 season. Differences between the RI lines with three Harbin 2-row QTL alleles and those with three Turkey 6 QTL alleles were statistically significant in both seasons $(t=2.62$ in the 2001 season and $t=$ 3.26 in the 2004 season). These three QTLs and flanking markers could become useful sources of FHB resistance in barley breeding programs.

We detected one of the most effective QTLs for FHB 
severity in the present study, because the QTL located in the vicinity of the $c l y 1 / C l y 2$ locus was found in two different genetic backgrounds of the highly resistant varieties, i.e. Russia 6 and Harbin 2-row, selected by Takeda and Heta (1989). This QTL may be very important to introgress resistance alleles into barley germplasm in breeding programs.

\section{Acknowledgements}

We thank Drs. D. Saisho, K. Tanno and Mr. M. Ishii, Res. Inst. Biores., Okayama Univ., for their invaluable technical advice. This research was supported by a grant from Core Research for Evolutional Science and Technology (CREST), Japan Science and Technology Corporation.

\section{Literature Cited}

Atanasoff,D. (1920) Fusarium-blight (scab) of wheat and other cereals. J. Agr. Res. 2: 1-32.

Ayoub,M. and D.E.Mather (2002) Effectiveness of selective genotyping for detection of quantitative trait loci: an analysis of grain and malt quality traits in three barley populations. Genome 45: 1116-1124.

Bai,G. and G.Shaner (1994) Scab of wheat: Prospects for control. Plant Disease 78: 760-766.

Basten,C.J., B.S.Weir and Z.B.Zeng (1994) Zmap-a QTL cartographer. In "The 5th World Congress on Genetic Applied to Livestock Production: Computing Strategies and Software, vol. 22" Smith, C. et al. (eds.) The Organizing Committee, 5th World Congress on Genetics Applied to Livestock Production, Ontario, Canada, p. 65-66 (http://statgen.ncsu.edu/qtlcart/ cartographer.html).

Chen, X.M., R.F.Line and H.Leung (1998) Genome scanning for resistance-gene analogs in rice, barley, and wheat by highresolution electrophoresis. Theor. Appl. Genet. 98: 345-355.

Costa,J.M., A.Corey, P.M.Hayes, C.Jobet, A.Kleinhofs, A.KopischObusch, S.F.Kramer, D. Kudrna, M.Li, O.Riera-Lizarazu, K.Sato, P.Szucs, T.Toojinda, M.I.Vales and R.I. Wolfe (2001) Molecular mapping of the Oregon Wolfe Barleys: a phenotypically polymorphic doubled-haploid population. Theor. Appl. Genet. 103: 415-424.

Dahleen, L.S., H.A. Agrama, R.D. Horsley, B.J. Steffenson, P.B. Schwarz, A.Mesfin and J.D.Franckowiak (2003) Identification of QTLs associated with Fusarium head blight resistance in Zhedar 2 barley. Theor. Appl. Genet. 108: 95-104.

de la Pena, R.C., K.P.Smith, F.Capettini, G.J.Muehlbauer, M.GalloMeagher, R.Dill-Macky, D.A.Somers and D.C.Rasmusson (1999) Quantitative trait loci associated with resistance to Fusarium head blight and kernel discoloration in barley. Theor. Appl. Genet. 99: 561-569.

Falconer,D.S. (1989) Introduction to quantitative genetics (third edition). Longman Group UK Ltd., London, UK. p. 160-183.

Hori,K., T.Kobayashi, A.Shimizu, K.Sato, K.Takeda and S.Kawasaki (2003) Efficient construction of high-density linkage map and its application to QTL analysis in barley. Theor. Appl. Genet. 107: 806-813.

Hori,K., T.Kobayashi, K.Sato and K.Takeda (2005) QTL analysis of Fusarium head blight resistance using a high-density linkage map in barley. Theor. Appl. Genet. 111: 1661-1672.

Kosambi,D.D. (1944) The estimation of map distance from recombi- nation values. Ann. Eugen. 12: 172-175.

Lander,E.S., P.Green, J.Abrahamson, A.Barlow and M.J.Daly (1987) MAPMAKER: An interactive computer package for constructing primary genetic linkage maps of experimental and natural populations. Genomics 1: 174-181.

Lander,E.S. and D.Botstein (1989) Mapping Mendelian factors underlying quantitative trait using RFLP linkage maps. Genetics 121: 185-199.

Ma,Z., B.J.Steffenson, L.K.Prom and N.L.Lapitan (2000) Mapping of quantitative trait loci for Fusarium head blight resistance in barley. Phytopathology 90: 1079-1088.

Mesfin, A., K.P.Smith, R.Dill-Macky, C.K.Evans, R.Waugh, C.D. Gustus and G.J.Muehlbauer (2003) Quantitative trait loci for Fusarium head blight resistance in barley detected in a tworowed by six-rowed population. Crop Sci. 43: 307-318.

Ramsay,L., M.Macaulay, S.degli Ivanissevich, K.MacLean, L.Cardle, J.Fuller, K.J.Edwards, S.Tuvesson, M.Morgante, A.Massari, E. Maestri, N.Marmiroli, T.Sjakste, M.Ganal, W.Powell and R. Waugh (2000) A simple sequence repeat-based linkage map of barley. Genetics 156: 1997-2005.

Steffenson, B.J., L.K.Prom, B.Salas, T.G.Fetch, D.M.Wesenberg and H.E.Bockleman (1996) Severity of Fusarium head blight and concentrations of deoxynivalenol in near-isogenic lines of barley differing for several agronomic characters. In "Proc. 7th Intl. Barley Genetics Symp.” Slinkard,A. et al. (eds.), University Extension Press, Saskatoon, Saskatchewan, p. 774-776.

Steffenson, B.J. (2003) Fusarium head blight of barley: impact, epidemics, management, and strategies for identifying and utilizing genetic resistance. In "Fusarium Head Blight of Wheat and Barley" Leonard,K.J. and W.R.Bushnell (eds.), American Pytopathology Press, St.Paul, MN, p. 241-295.

Takeda,K. and H.Heta (1989) Establishing the testing method and a search for the resistant varieties to Fusarium head blight in barley. Jpn. J. Breed. 39: 203-216.

Tanno,K., S.Taketa, K.Takeda and T.Komatsuda (2002) A DNA marker closely linked to the vrs 1 locus (row-type gene) indicates multiple origins of six-rowed cultivated barley (Hordeum vulgare L.). Theor. Appl. Genet. 104: 54-60.

Turuspekov, Y., Y.Mano, I. Honda, N. Kawada, Y. Watanabe and T.Komatsuda (2004) Identification and mapping of cleistogamy genes in barley. Theor. Appl. Genet. 109: 480-487.

Ukai,Y., R.Ohsawa, A.Saito and T.Hayashi (1995) MAPL: A package of computer programs for construction of DNA polymorphism linkage maps and analysis of QTL. Breed. Sci. 45: 139-142 (http://wheat.ab.a.u-tokyo.ac.jp/ ukai/).

Vos,P., R.Hogers, M.Bleeker, M.Reijan, T.van de Lee, M.Hornes, A.Frijters, J.Pot, J.Peleman, M.Kuiper and M.Zabeau (1995) AFLP: a new technique for DNA fingerprinting. Nucleic Acid Res. 23: 4407-4414.

Yoshida,M., N.Kawada and T.Tohnooka (2005) Effect of row type, flowering type and several other spike characters on resistance to Fusarium head blight in barley. Euphytica 141: 217-227.

Zhou,X., M.Chao and X.Liang (1991) Screening and testing of barley varieties for scab resistance. Acta. Phytophylacia. Sin. 18: 261264.

Zhu,H., L.Gilchrist, P.Hayes, A.Kleinhofs, D.Kudrna, Z.Liu, L.Prom, B.Steffenson, T.Toojinda and H.Vivar (1999) Does function follow form? Principal QTLs for Fusarium head blight (FHB) resistance are coincident QTLs for inflorescence traits and plant height in a doubled haploid population of barley. Theor. Appl. Genet. 99: 1221-1232. 